\title{
THE INFLUENCE OF NUCLEATION AND DROPLET GROWTH ON THE ONSET OF CONDENSATION IN SUPERSONIC NOZZLE EXPANSIONS
}

\author{
Gerald Wilemski, Barbara E. Wyslouzil, Mitzi G. Beals, and Michael B. Frish
}

\section{INTRODUCTION}

Unlike most other techniques used to study nucleation, supersonic nozzles do not yield nucleation rates directly because the length of time over which nucleation contributes significantly to particle formation is not easy to determine or control. Nevertheless, experiments in nozzles are extremely important because they provide higher rates of cooling, higher supersaturations and higher nucleation rates than any of the other techniques. Their operating conditions are more typical of important industrial conditions found in aerodynamic and turbomechanical flows where homogeneous condensation can have serious consequences for the gas flow behavior. Because the fluid mechanics of nozzles is well-understood, condensation experiments in the nozzle are amenable to sophisticated modeling efforts, and much useful insight can be gained regarding the nucleation and droplet growth processes under these severe cooling conditions.

Because of the difficulty in measuring rates directly, nozzle experiments have tended to emphasize measurements of the actual density or pressure profiles during flow. A key piece of information derived from these measurements is the onset of condensation. The onset of condensation is the point in the expanding flow where the density, pressure, and temperature deviate significantly from the isentropic values. One of the most important issues is to understand the variation of the observed onset of condensation with different experimental conditions. There are many variables that can affect this behavior. These include the cooling rate and the supply conditions: pressures of the carrier gas and condensible vapor and the stagnation temperature. Also, the nature of the carrier gas may potentially play a role. Many studies have been made, ${ }^{1,2}$ but many poorly understood findings persist. Examples can be readily cited for the condensation of water, ${ }^{3}$ argon, ${ }^{4}$ and nitrogen. ${ }^{5}$

Less attention has been paid to the more microscopic question of the character of the condensate at onset. Questions regarding the size and concentration of the growing condensate particles at onset are much harder to answer experimentally and have not received intensive theoretical scrutiny either, although the means to do so has long existed. ${ }^{6}$ While nucleation always initiates condensation, it does not follow that the onset of condensation is necessarily due to nucleation alone. Nucleation dominated onset has been identified by Wegener, Clumpner, and $\mathrm{Wu}^{7}$ in the case of ethanol condensation. However, for other materials or even for ethanol under other conditions, it is possible that droplet growth may contribute significantly, even predominantly to the amount of condensate at onset. 
This paper summarizes our recent experimental work using a gently diverging supersonic Laval nozzle to investigate the variation of onset temperature and pressure for varying amounts of condensible vapor in an excess of carrier gas. Many similar studies have been carried out previously, but the results of these studies are usually not sufficiently well documented to enable us to do modeling studies that permit assessment of the condensate characteristics at onset. By carrying out modeling of the particle size distributions for our own experiments, we can avoid this difficulty. In modeling our experimental results, we have found that the mechanism for producing observable condensate varies considerably with conditions. Nucleation of small droplets can dominate at one extreme, but droplet growth can also be found to play a dominant role at other conditions.

Following a brief description of our experimental apparatus and methodology in Section II., we present our experimental results in Section III. and compare them with results from related experiments. In Section IV. we describe our modeling procedure, present the calculated size distributions at onset, and discuss the implications of these results.

\section{EXPERIMENTAL APPARATUS AND DATA ANALYSIS}

The experimental apparatus and data analysis were described in detail in a recent publication ${ }^{8}$ and are reviewed only briefly here. As illustrated in Figure 1, the apparatus consists of an intermittent, low Mach number, supersonic Laval nozzle equipped with a Mach-Zender interferometer. The desired amount of condensible vapor is accurately metered from a pressurized saturator into a previously evacuated supply tank. The required amount of $\mathrm{N}_{2}$ carrier gas is then added to complete the mixture. The nozzle is two dimensional with a $0.5 \times 1.23 \mathrm{~cm}^{2}$ throat, a $7.9 \mathrm{~cm}$ long supersonic portion and an exit-to-throat area ratio of 1.37 that yields a maximum Mach number of 1.72 for a diatomic gas. During a $300 \mathrm{~ms}$ run, gas from a heated plenum flows through the nozzle into an evacuted dump tank, and steady one dimensional density, temperature and pressure gradients are established. An analysis of the interference fringes measured during the steady flow period, yields the relative density profiles, $\rho / \rho_{0}$, as a function of position in the nozzle. The relative density profile for a non-condensing flow follows that described by the one-dimensional adiabatic gas dynamics equations and is used to accurately calibrate the shape of the nozzle. This calibration is critical in a small nozzle because boundary layers significantly alter the effective shape of the nozzle and are a strong function of the pressure. The relative density profiles for the flow of a condensing gas mixture together with the diabatic gas dynamics equations yield the temperature, pressure and condensed mass profiles. 


\section{DISCLAIMER}

Portions of this document may be illegible in electronic image products. Images are produced from the best available original document. 


\section{EXPERIMENTAL RESULTS}

We conducted condensation experiments over a wide range of initial water, ethanol, and propanol concentrations. these experiments were conducted using stagnation pressures of 2.5 or 3.0 atmospheres. These pressures provide clean interferograms while still letting us observe onset even at relatively low condensible vapor concentrations. When the stagnation pressure is very high the heat released by the condensing vapor may not be enough to perturb the flow noticibly. When the stagnation pressure is too low, interferometry becomes difficult because the absolute changes in the gas density are small.

As shown elsewhere, ${ }^{8,9}$ the onset of condensation often occurs after the condensing flow curve has already begun to deviate somewhat from the theoretical isentrope for the wet flow, the wet isentrope. Depending on how rapidly this deviation occurs, there is some uncertainty in determining the onset of condensation. ${ }^{10}$ To reduce this ambiguity, we define the onset of condensation as the point where the temperature on the condensing flow curve first deviates from the temperature of the wet isentrope by $1 \mathrm{~K}$. As discussed more extensively in our previous paper, ${ }^{8}$ this definition is not unique but most reasonable definititions of the onset of condensation will be quite close to this one.

Experiments at constant stagnation pressure with varying condensible vapor pressure confirm that our supersonic nozzle results are consistent with those of other workers generated using a variety of experimental techniques. Our results for water are shown in Figure 2. where they are compared with data of Pouring, ${ }^{11}$ Roberts, ${ }^{12}$ Stein and Moses, ${ }^{13}$, and Stein. ${ }^{14}$ The results for ethanol are shown in Figure 3., where they are compared with those of Wegener, Clumpner, and $\mathrm{Wu},{ }^{7}$ Dawson et al. ${ }^{15}$ Peters, ${ }^{16}$ Franck and Hertz, ${ }^{17}$ and Katz and Ostermeier. ${ }^{18}$ Finally our results for n-propanol are shown in Figure 4. along with shock tube results of Peters, ${ }^{16}$ since no other nozzle data exist for this species. Figures 2 and 3 have been published previously ${ }^{8}$ but are included for completeness. Experimental onset and starting conditions are summarized in in Tables I., II., and III. for, respectively, water, ethanol, and n-propanol. The results from these uniary experiments agree well with experimental results from other nozzles and shock tubes.

\section{CONDENSATION MODELING AND DISCUSSION}

Theoretical calculations of condensation in the nozzle were performed using the integral steady state (ISS) model developed by Oswatitsch ${ }^{6}$ to simulate the nucleation and growth of particles. Our version of this model includes the effects of heat addition to the flow through the diabatic gasdynamics equations. ${ }^{2}$ The model is based on the classical kinetic model of cluster formation which considers cluster size to change only by monomer condensation and evaporation. The ISS method uses the steady state nucleation rate $J$ to compute the number density $\Delta N$ of new particles formed at each point $x$ in the expansion from the conservation law, $\Delta N=(J / u) \Delta x$; 
$u$ is the local flow velocity. With an appropriate droplet growth law the condensate mass fraction can be calculated versus $x$, and from this the change in flow properties can be obtained by integrating the diabatic flow equations. This approach has been used extensively for simulating condensation in nozzles. ${ }^{2}$ In these calculations, the classical isothermal steady state rate expression, $J_{c l}$, was used for $J$. Following conventional practice, ${ }^{7}$ a multiplicative adjustment factor $\Gamma$ was used to bring the calculated and measured values of the onset temperature into agreement. Thus $J$ is expressed as $J=\Gamma J_{\mathcal{c l}}$. In general, $\Gamma$ varies with experimental conditions, but for a given expansion it is a temperature independent constant.

Figures 5. and 6 . contain the results of a modeling calculation for $1 \%$ water vapor in nitrogen at a total stagnation pressure of 2.5 atm. In Fig. 5, calculated and measured temperature profiles for the condensing flow are shown along with the isentropic behavior in the absence of condensation. In Fig. 6, calculated number densities of condensate particles are plotted as a function of particle radius at onset, $t=18.2 \mu$ s downstream of the nozzle throat, where only $1.7 \%$ of the water has condensed, and at a position much farther downstream, $t=42.7 \mu \mathrm{s}$, where $80.4 \%$ of the water is calculated to have condensed. Besides the ISS model results, each figure contains calculations based on a much more detailed discrete-sectional (DS) model described elsewhere. ${ }^{20}$ The DS results are included as a check on the accuracy and reliability of the much simpler ISS results.

The ISS method produces results very close to those of the DS model provided that both the steady state nucleation rate expression and droplet growth law used are consistent with the forms of the forward and reverse rate coefficients used in the DS model. The ISS model results in Figs. 5 and 6 were obtained using the quasi-steady isothermal droplet growth law including evaporation, ${ }^{7}$ and droplet growth was started at a size $1 \%$ greater than critical size. The temperature profiles indicate that droplet growth is overestimated, but the onset region is well described. The particle size distributions at onset, $x=-0.2$ and $t=18.2 \mu \mathrm{s}$, and further downstream are in good agreement These theoretical results indicate that at onset most of the condensed mass is already present in particles much larger than the critical size $(0.5 \mathrm{~nm})$ and that substantial droplet growth has already occurred. A second set of comparisons between experiment and the ISS model is shown in Fig. 7-9. These experiments used a stagnation pressure of $3 \mathrm{~atm}$, a lower initial water vapor pressure, and a lower stagnation temperature. These changes produced a considerable decrease in onset temperature from $244 \mathrm{~K}$ to $220 \mathrm{~K}$. These experiments were modeled with an improved droplet growth law based on Young's ${ }^{21}$ work as simplified by Peters and Paikert. ${ }^{22}$ This growth law covers the transition and continuum flow regimes as well as the more relevant free molecular flow condition for our experiments. The growth law also accounts for droplet heating during growth (or evaporative cooling) permitting more realistic modeling of post-onset flow. These results are very similar to those of the previous case. Figure 9 
shows clearly that essentially none of the condensed mass is found in critically sized particles. About $85 \%$ of the condensed mass is found in particles with radii of at least $5 \mathrm{~nm}$, i.e. at least 1000 times the volume of a critical nucleus. Droplet growth obviously accounts for the condensed mass at onset in these two cases.

This behavior contrasts starkly with the view, developed for low temperature ethanol condensation, that at onset most of the condensed mass exists as a cloud of tiny $(0.5 \mathrm{~nm})$ near-critical nuclei with droplet growth contributing substantially only after the peak nucleation zone. ${ }^{7}$ To explore this dramatic divergence of views, we modeled several of our ethanol experiments and compared them to modeling results for the conditions of Wegener et al. ${ }^{7}$ using our nozzle shape, since theirs was not readily available.

As opposed to the above water results, ethanol condensation calculations for the conditions used by Wegener et al. ${ }^{7}$ yield results supporting the picture of decoupled nucleation and growth. These results are shown in Figures 10 and 11. The size distribution at onset is very sharp, criticallysized particles dominate, and most of the mass lies in the smallest sizes. It should be noted this behavior is a consequence of the exceedingly high nucleation rates achieved in the experiments of Wegener et al.. ${ }^{7}$ In calculations simulating our ethanol condensation experiments, we observe a transition from this decoupled behavior to a regime in which nucleation and droplet growth are equally important as for the case of water condensation. Figure 10 also shows the very broad ethanol size distribution computed for one set of our conditions. Figure 11 compares cumulative mass distribution curves illustrating this transition. The sharpest distribution corresponds to the conditions of Wegener et al.; the other two curves span the temperature range covered by our experiments. We conclude that the magnitude of nucleation rate achievable under different conditions has an major effect on the relative importance of nucleation and droplet growth at onset.

Finally, we performed calculations for several of our propanol experiments. The results shown in Figs. 12-15 are for a low (4 Torr) and intermediate ( 8 Torr) initial propanol pressure. The droplet growth law of Young, ${ }^{21}$ and Peters and Paikert ${ }^{22}$ was used for these calculations. The calculated temperature profiles shown in Figs. 12 and 13 are in reasonably good agreement with the curves derived from experiment. In Figs. 14 and 15 we see behavior similar to that found for ethanol. The size distribution at onset for the lower condensible pressure and lower onset temperature is narrower and more mass is found in smaller particle sizes than for the higher condensible pressure case.

\section{SUMMARY AND CONCLUSIONS}

At the onset of condensation in a supersonic nozzle expansion, gas flow properties begin to deviate from their isentropic values. The latent heat released by condensation heats the flow and causes the pressure and density to increase. While nucleation always initiates condensation, the 
onset of condensation is not necessarily due to nucleation alone. Our modeling results, indicate that the mechanism for producing observable condensate varies considerably with conditions. The particle size distribution at onset appears to undergo a continuous transition from narrow to broad as the onset temperature and pressure increase. When condensible vapor at a low partial pressure expands to a low temperature, nucleation rates are very high at onset, and the mass of condensate occurs mainly as droplets of near critical size. Droplet growth contributes substantially to the accumulation of condensate only after nucleation subsides. At high pressures of condensible vapor, onset occurs at higher temperatures and lower supersaturations. The condensate mass at onset then occurs overwhelmingly as very large supercritical droplets. While nucleation continues at an appreciable rate in the onset region, freshly nucleated droplets contribute negligibly to the total condensed mass.

\section{ACKNOWLEDGMENT}

This work was supported by the United States Department of Energy, Division of Engineering and Geosciences, Office of Basic Energy Sciences, under Contract No. DE-AC02-84ER13154 and Grant No. DE-FG02-92ER14257.

\section{DISCLAIMER}

This report was prepared as an account of work sponsored by an agency of the United States Government. Neither the United States Government nor any agency thereof, nor any of their employees, makes any warranty, express or implied, or assumes any legal liability or responsibility for the accuracy, completeness, or usefulness of any information, apparatus, product, or process disclosed, or represents that its use would not infringe privately owned rights. Reference herein to any specific commercial product, process, or service by trade name, trademark, manufacturer, or otherwise does not necessarily constitute or imply its endorsement, recommendation, or favoring by the United States Government or any agency thereof. The views and opinions of authors expressed herein do not necessarily state or reflect those of the United States Government or any agency thereof. 


\section{References}

${ }^{1}$ Yu. A. Ryzhov, U.G. Pirumov, and V.N. Gorbunov, Nonequilibrium Condensation in HighSpeed Gas Flows (Gordon and Breach, New York, 1989).

${ }^{2}$ P.P. Wegener and B.J.C. Wu, Adv. Colloid. Interface Sci. 7, 325 (1977).

${ }^{3}$ D. Barschdorff, Phys. Fluids 18, 529 (1975).

4 J. Steinwandel and T. Buchholz, Aerosol Sci. and Tech. 3, 71 (1984).

5 J. Steinwandel, Ber. Bunsenges. Phys. Chem. 89, 481 (1985).

${ }^{6}$ K. Oswatitsch, Z. Angew. Math. Mech. 22, 1 (1942).

${ }^{7}$ P.P. Wegener, J.A. Clumpner, and B.J.C. Wu, Phys. Fluids 15, 1869 (1972).

${ }^{8}$ B.E. Wyslouzil, G. Wilemski, M.G. Beals and M. Frish, Phys. Fluids 6, 2845 (1994).

${ }^{9}$ B.E. Wyslouzil, M.G. Beals and G. Wilemski, see Appendix C, this report.

${ }^{10}$ H.S. Stever, in Fundamentals of Gas Dynamics, edited by H.W. Emmons (Princeton Univ. Press, Princeton, 1958), pp.526-573.

${ }^{11}$ A.A. Pouring, Ph.D. Thesis, Yale University, New Haven CT (1963).

${ }^{12}$ R. Roberts, Report No. 97, MIT, Gas Turbine Lab, Cambridge MA (1969).

${ }^{13}$ G.D. Stein and C.A. Moses, J. Colloid Interface Sci. 39, 504 (1972).

${ }^{14}$ G.D. Stein, Ph.D. Thesis, Yale University, New Haven CT (1967).

15 D.B. Dawson, E.J. Willson, P.G. Hill, and K.C. Russell, J. Chem. Phys. 51, 5389 (1969).

${ }^{16}$ F. Peters, J. Chem. Phys. 77, 4788 (1982).

${ }^{17}$ J.P. Franck and H.G. Hertz, Z. Physik 143, 559 (1956).

${ }^{18}$ J.L. Katz and B.J. Ostermeier, J. Chem. Phys. 47, 478 (1967).

19 F.F. Abraham, Homogeneous Nucleation Theory (Academic, New York, 1974).

${ }^{20}$ G. Wilemski, B.E. Wyslouzil, M. Gauthier, and M.B. Frish, in Proceedings of the Eleventh

Symposium on Energy Engineering Sciences, Argonne IL, May 1993 (NTIS CONF-9305134) (1993)

p. 56.

${ }^{21}$ J.B. Young, Int. J. Heat Mass Transfer 36, 2941 (1993).

${ }^{22}$ F. Peters and B. Paikert, Int. J. Heat Mass Transfer 37, 293 (1994). 
Table 1. Summary of Experimental Conditions for Water Vapor.

\begin{tabular}{|c|c|c|c|c|}
\hline \multicolumn{5}{|c|}{ Table 1: Summary of Experimental Conditions: Water } \\
\hline Po (atm) & Pc (Torr) & To (K) & T (K) & P \\
\hline 3 & 17.22 & 316 & 233.7 & 5.94 \\
\hline 3 & 17.22 & 316 & 233.6 & 5.92 \\
\hline 3 & 17.22 & 316 & 232.5 & 5.85 \\
\hline 2.5 & 18 & 316 & 239.1 & 6.66 \\
\hline 2.5 & 15 & 316 & 235.6 & 5.32 \\
\hline 2 & 20.13 & 316 & 242 & 7.83 \\
\hline 2 & 20.13 & 316 & 240.3 & 7.6 \\
\hline 2 & 20.13 & 316 & 236.7 & 6.88 \\
\hline 2 & 20.13 & 306 & 236.7 & 8.1 \\
\hline 2 & 19.13 & 316 & 234.8 & 6.03 \\
\hline 2 & 17.22 & 316 & 234.1 & 6.01 \\
\hline 2 & 17.22 & 316 & 233.3 & 5.9 \\
\hline 2 & 17.22 & 316 & 232.8 & 5.85 \\
\hline 2 & 17.22 & 316 & 232.8 & 5.9 \\
\hline 2 & 15.22 & 316 & 230.8 & 5.01 \\
\hline 1 & 17.22 & 316 & 238.2 & 6.32 \\
\hline 1 & 17.22 & 316 & 235.8 & 6.1 \\
\hline 1 & 17.22 & 316 & 234.2 & 5.96 \\
\hline 1 & 17.22 & 316 & 234 & 5.98 \\
\hline 1 & 17.22 & 316 & 234 & 5.89 \\
\hline 0.5 & 17.22 & 316 & 236 & 6.21 \\
\hline 0.5 & 17.22 & 316 & 236 & 6.2 \\
\hline 0.5 & 17.22 & 316 & 235.8 & 6.09 \\
\hline 0.5 & 17.22 & 316 & 235 & 6.15 \\
\hline 0.5 & 17.22 & 316 & 235 & 6.07 \\
\hline 0.5 & 17.22 & 316 & 234.8 & 6.04 \\
\hline 0.5 & 17.22 & 316 & 234 & 6 \\
\hline & & & & \\
\hline
\end{tabular}


Table 2. Summary of Experimental Conditions for Ethanol Vapor.

\begin{tabular}{|c|c|c|c|c|}
\hline \multicolumn{6}{|c|}{ Table 2: Summary of Experimental Conditions: Ethanol } \\
\hline Po (atm) & Pc (torr) & To (K) & T (K) & P \\
\hline 3 & 22.8 & 316 & 242.5 & 8.88 \\
\hline 3 & 20.8 & 316 & 240.5 & 7.86 \\
\hline 3 & 18.8 & 316 & 236.6 & 6.72 \\
\hline 3 & 16.8 & 316 & 235.2 & 5.88 \\
\hline 3 & 12.8 & 316 & 233.6 & 4.38 \\
\hline 3 & 12.8 & 316 & 233.3 & 4.36 \\
\hline 3 & 12.8 & 316 & 230.8 & 4.21 \\
\hline 3 & 10.8 & 310 & 228.6 & 3.67 \\
\hline 3 & 7.6 & 306 & 225.6 & 2.58 \\
\hline 3 & 5 & 299 & 216.6 & 1.6 \\
\hline 2.5 & 12.4 & 316 & 238 & 4.47 \\
\hline 2.5 & 7 & 316 & 225.2 & 2.82 \\
\hline 2 & 12.8 & 316 & 235.6 & 4.5 \\
\hline 2 & 12.8 & 316 & 235 & 4.47 \\
\hline 1 & 12.8 & 316 & 238.4 & 4.65 \\
\hline 1 & 12.8 & 316 & 237.5 & 4.6 \\
\hline 1 & 12.8 & 316 & 235 & 4.43 \\
\hline & & & & \\
\hline
\end{tabular}

Table 3. Summary of Experimental Conditions for Propanol Vapor.

\begin{tabular}{|c|c|c|c|c|}
\hline \multicolumn{5}{|c|}{ Table 3: Summary of Experimental Conditions: Propanol } \\
\hline Po (atm) & Pc (torr) & To (K) & T (K) & P \\
\hline 2.5 & 11 & 316 & 254.7 & 5.14 \\
\hline 2.5 & 10 & 310 & 251 & 4.72 \\
\hline 2.5 & 9.85 & 316 & 244.5 & 3.95 \\
\hline 2.5 & 8 & 316 & 242.4 & 3.34 \\
\hline 2.5 & 8 & 310 & 242.2 & 3.1 \\
\hline 2.5 & 6 & 310 & 237.6 & 2.34 \\
\hline 2.5 & 5 & 316 & 239.1 & 1.86 \\
\hline 2.5 & 4.5 & 310 & 232.1 & 1.62 \\
\hline 2.5 & 4 & 310 & 229.5 & 1.38 \\
\hline 2.5 & 3 & 310 & 225.2 & 0.97 \\
\hline
\end{tabular}




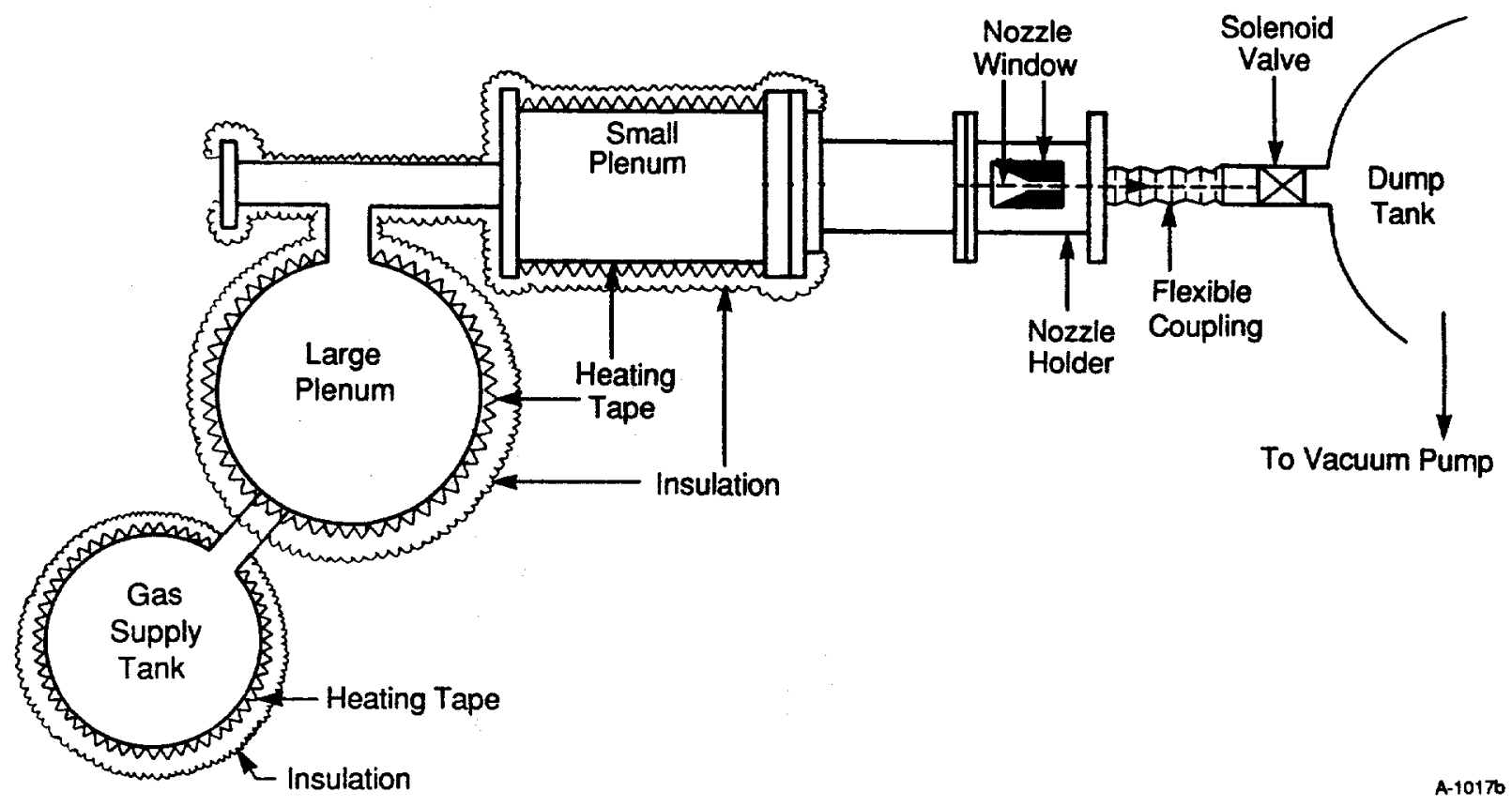

Figure 1. Schematic diagram of the supersonic nozzle flow system.

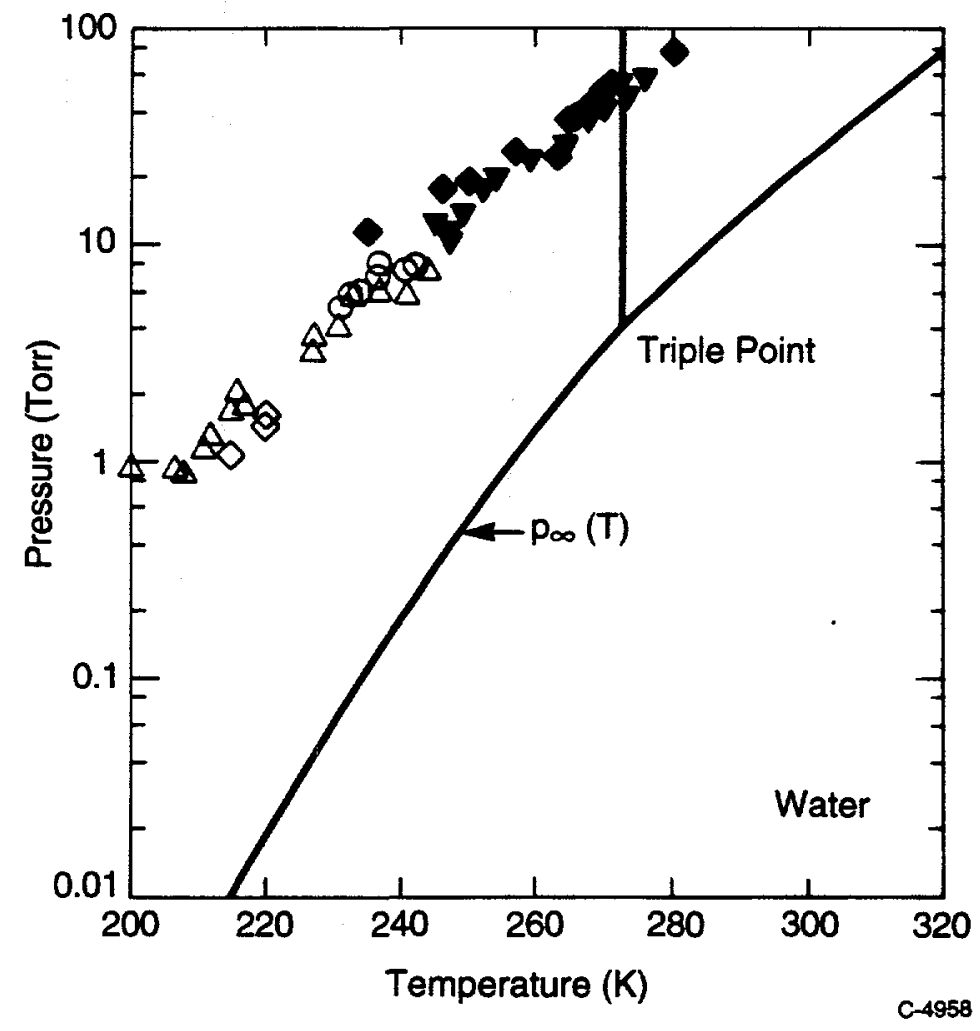

Figure 2. Onset of Water Condensation. Open circles, present work. Open triangles, Ref (11). Filled triangles, Ref (12). Filled diamonds, Ref (13). Open diamonds, Ref (14). 


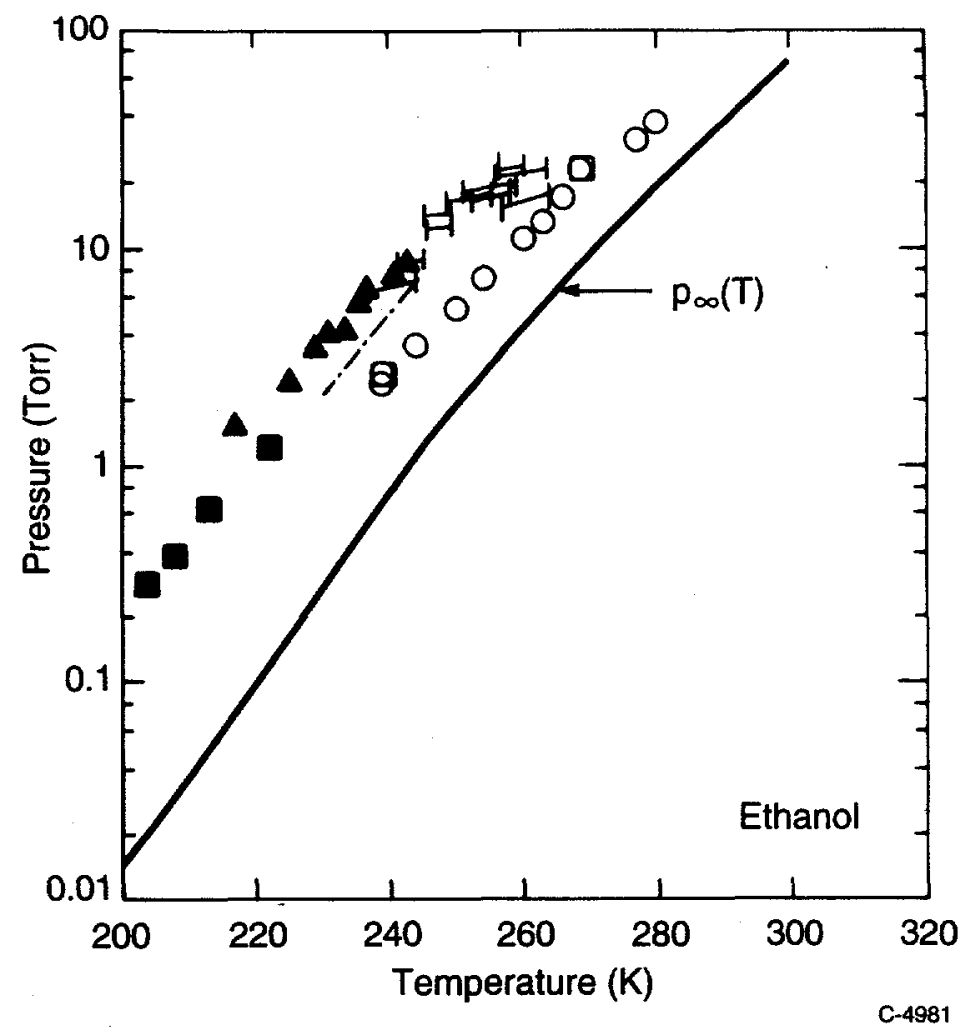

Figure 3. Onset of Ethanol Condensation. Filled triangles, present work. Filled squares, Wegener et al. (Ref. 7). Horizontal lines, Dawson et al. (Ref. 15). Dashed line, Peters (Ref. 16). Open circles, Frank and Hertz (Ref. 17).

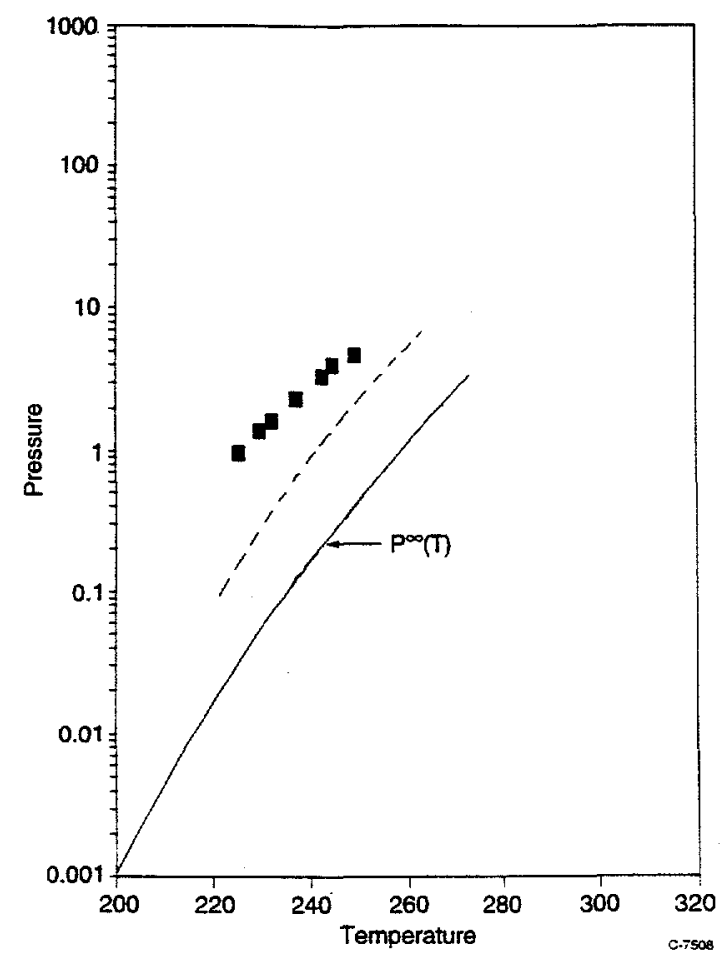

Figure 4. Onset of Propanol Condensation. The filled squares are the present work. The dashed line is the best fit through Peter's data, (Ref. 16). 


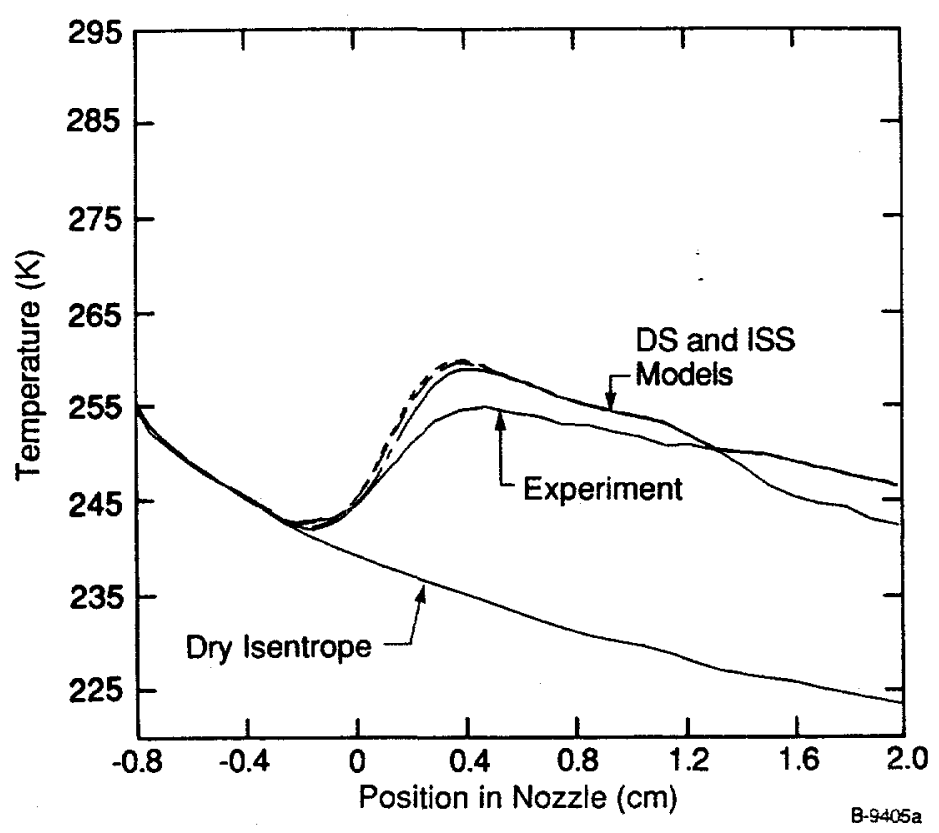

Figure 5. Experimental and Calculated Temperature Profiles for Water Condensation in the Supersonic Nozzle. $\mathrm{P}_{\mathrm{w}}=19.13$ Torr.

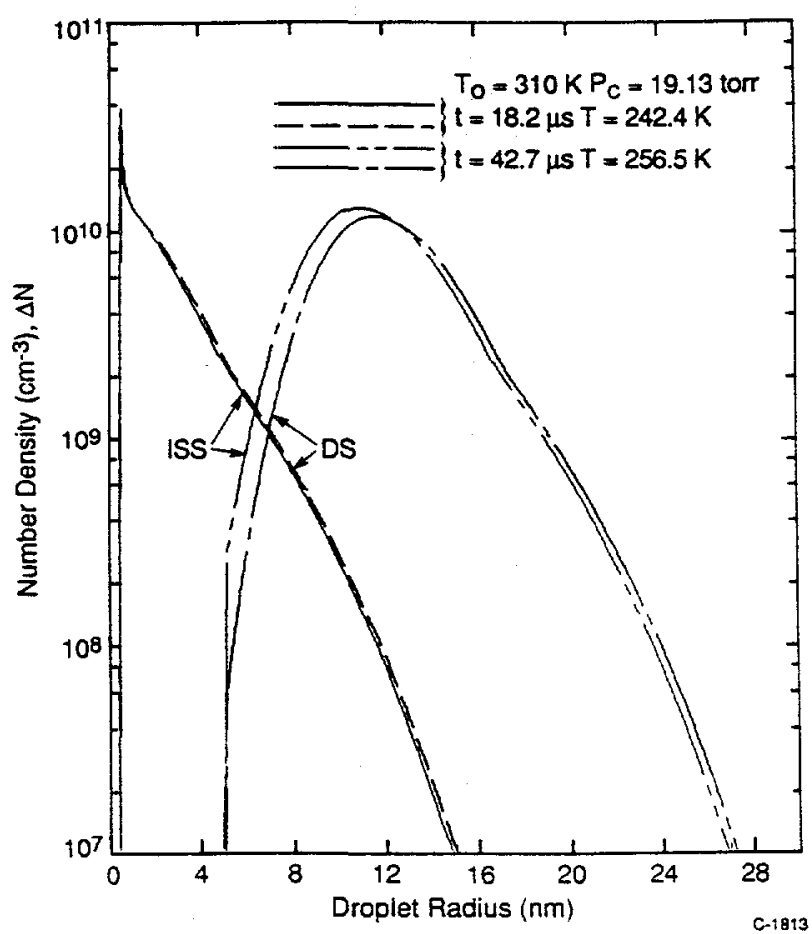

Figure 6. Comparison of Particle Size Distributions at Onset and as Condensation Nears Completion. Results were calculated using the discrete-sectional (DS) cluster kinetics model and an integral steady state (ISS) model with a quasi-steady isothermal droplet growth law including evaporation. 


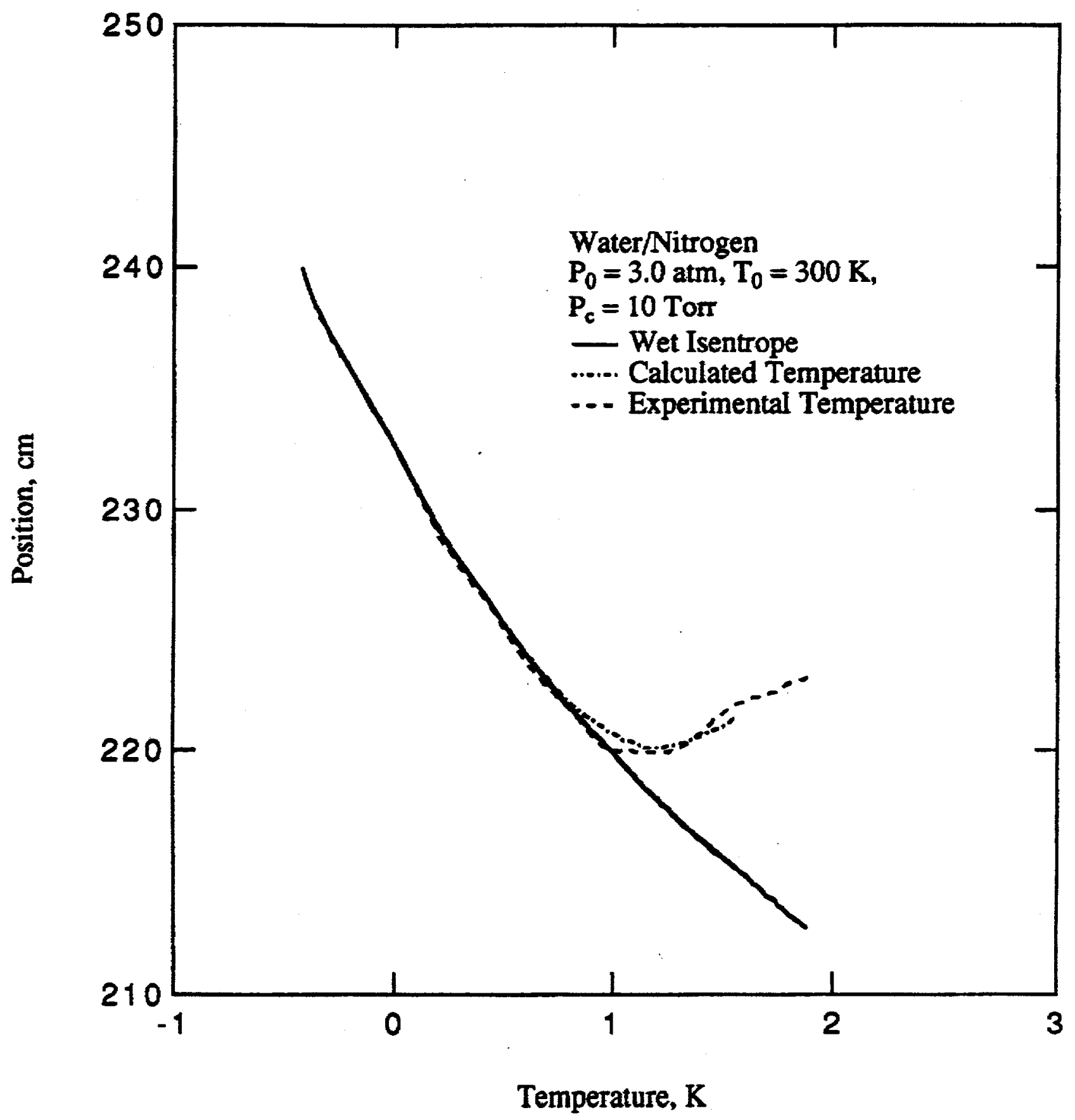

Figure 7. Experimental and Calculated Temperature Profiles for Water Condensation in the Supersonic Nozzle. $P_{c}=10$ Torr. 


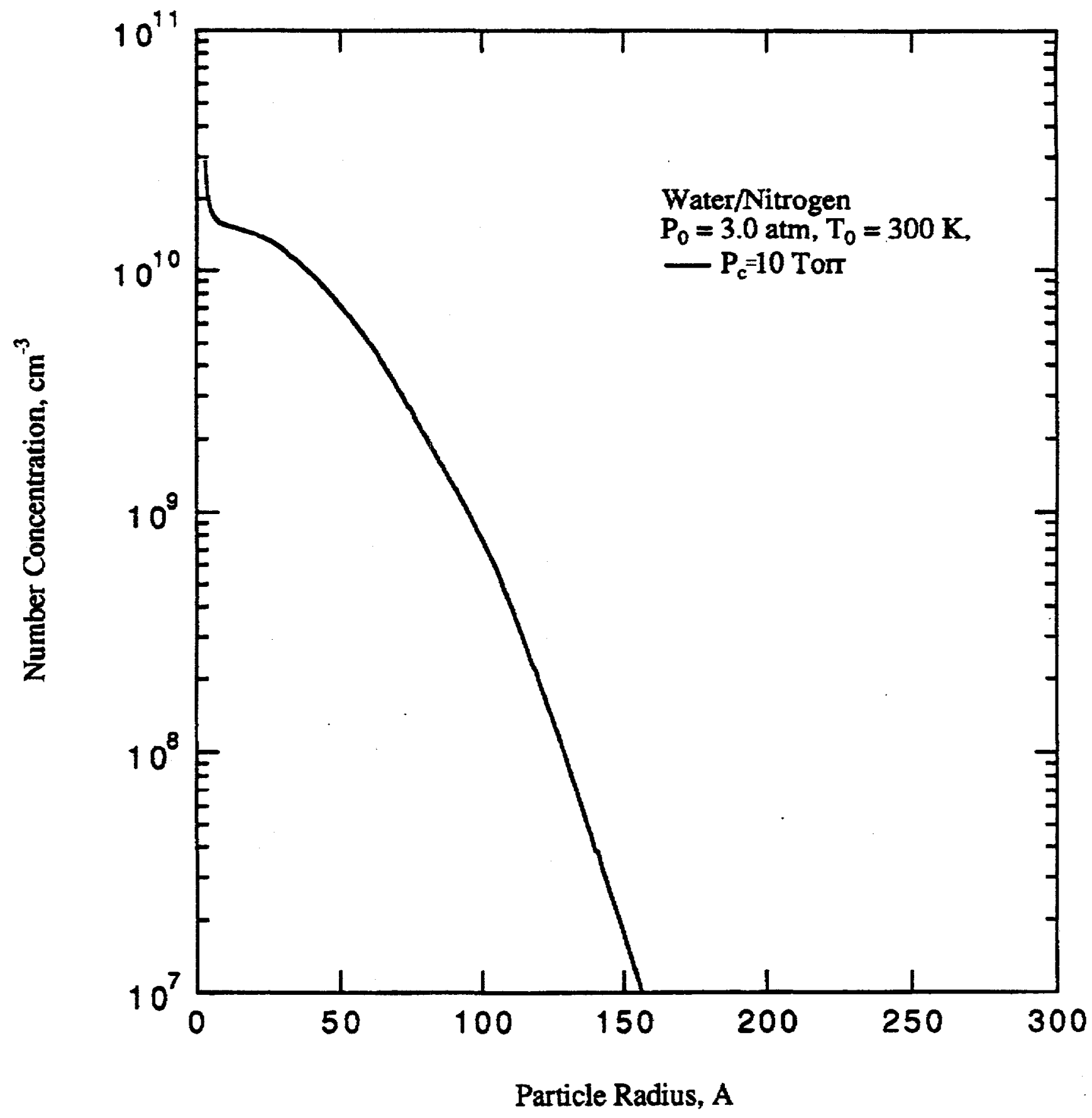

Figure 8. Predicted Particle Size Distribution at the Onset of Water Condensation in the Supersonic Nozzle. $P_{c}=10$ Torr. 


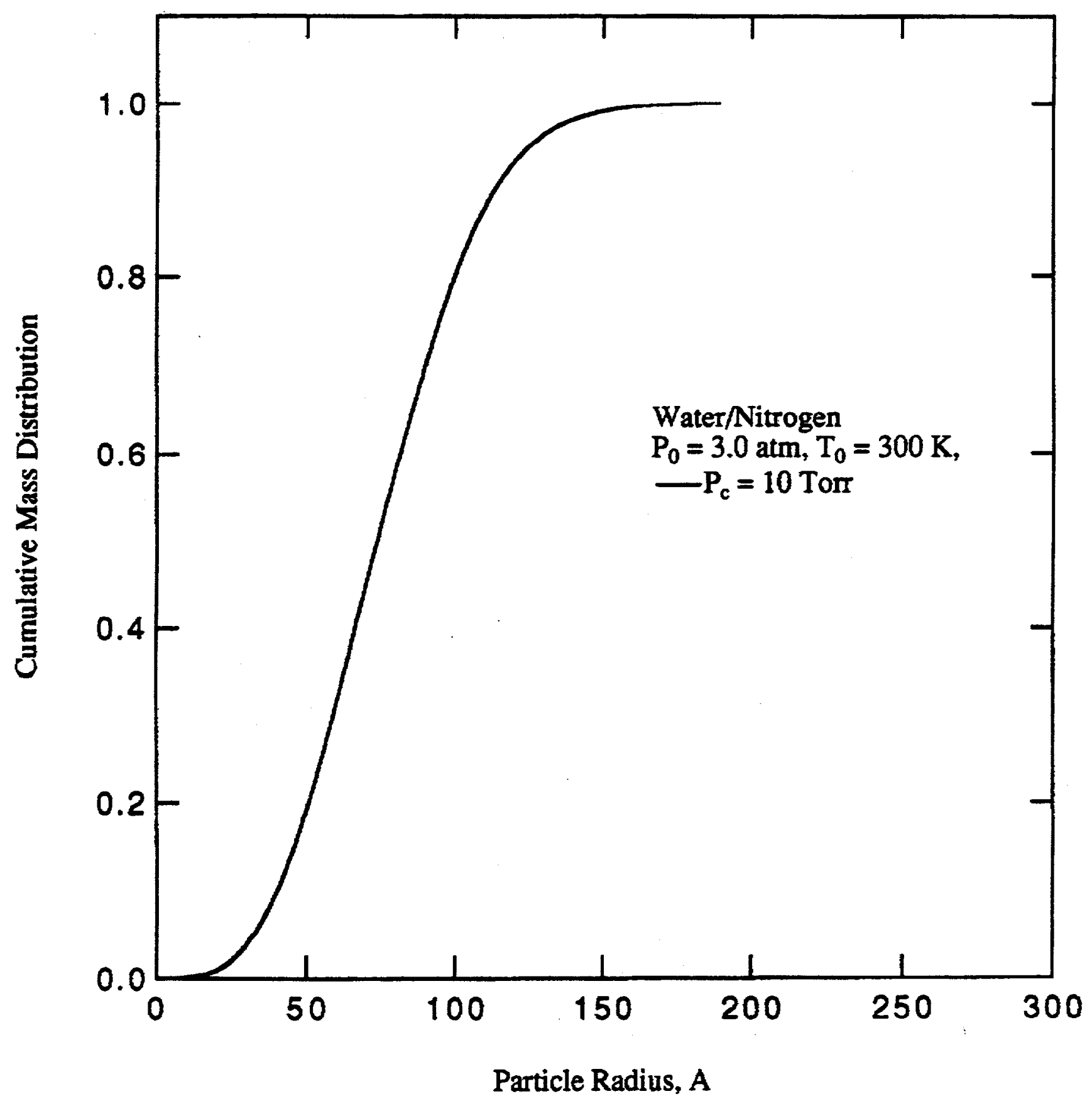

Figure 9. Predicted Mass Distribution at the Onset of Water Condensation in the Supersonic Nozzle. $\mathrm{P}_{\mathrm{c}}=10$ Torr. 


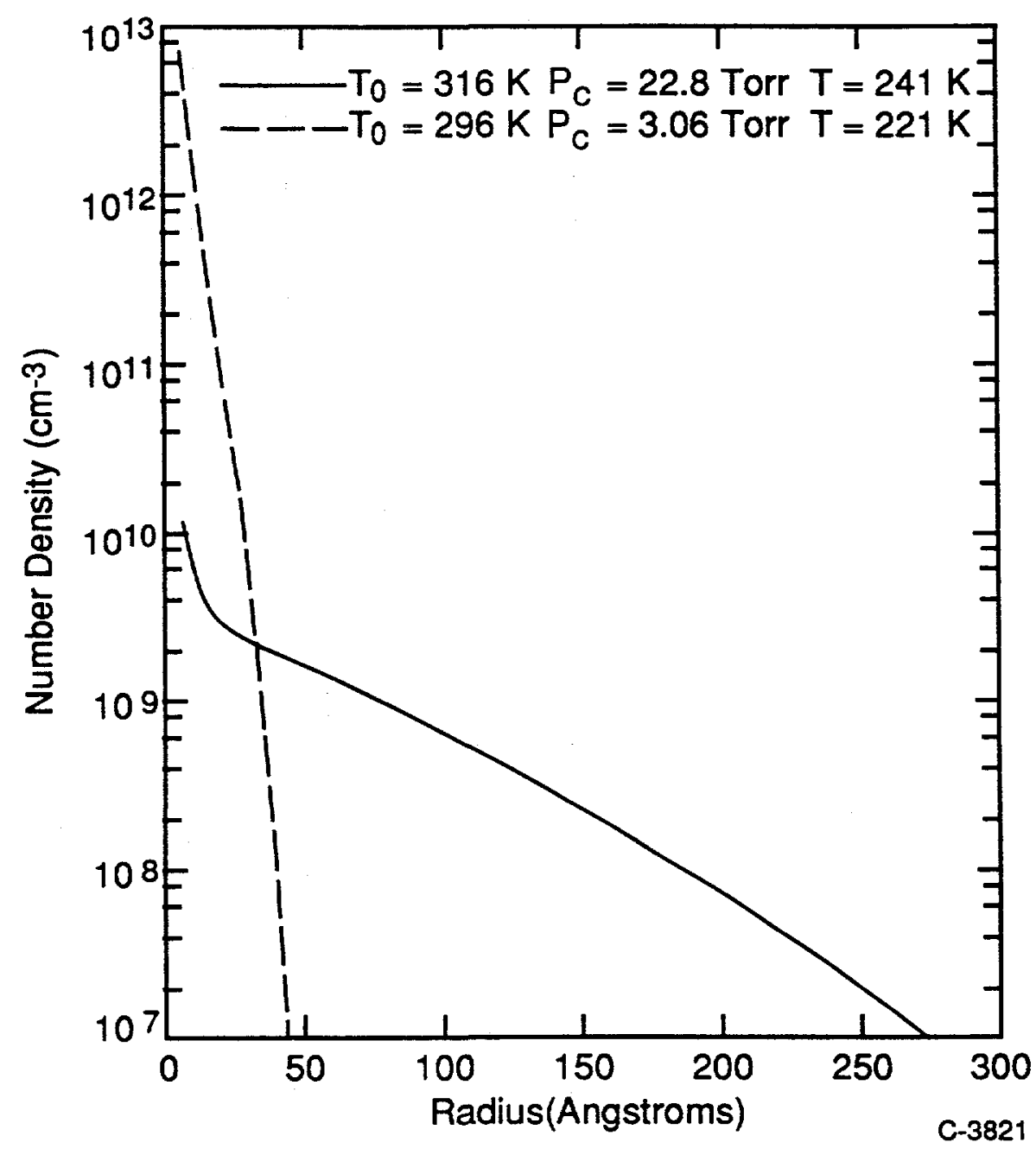

Figure 10. Ethanol Droplet Size Distributions at Condensation Onset for Low Temperature Conditions used by Wegener et al. (Ref. 7), ----, and Higher Temperature Conditions of the Present Work, - 


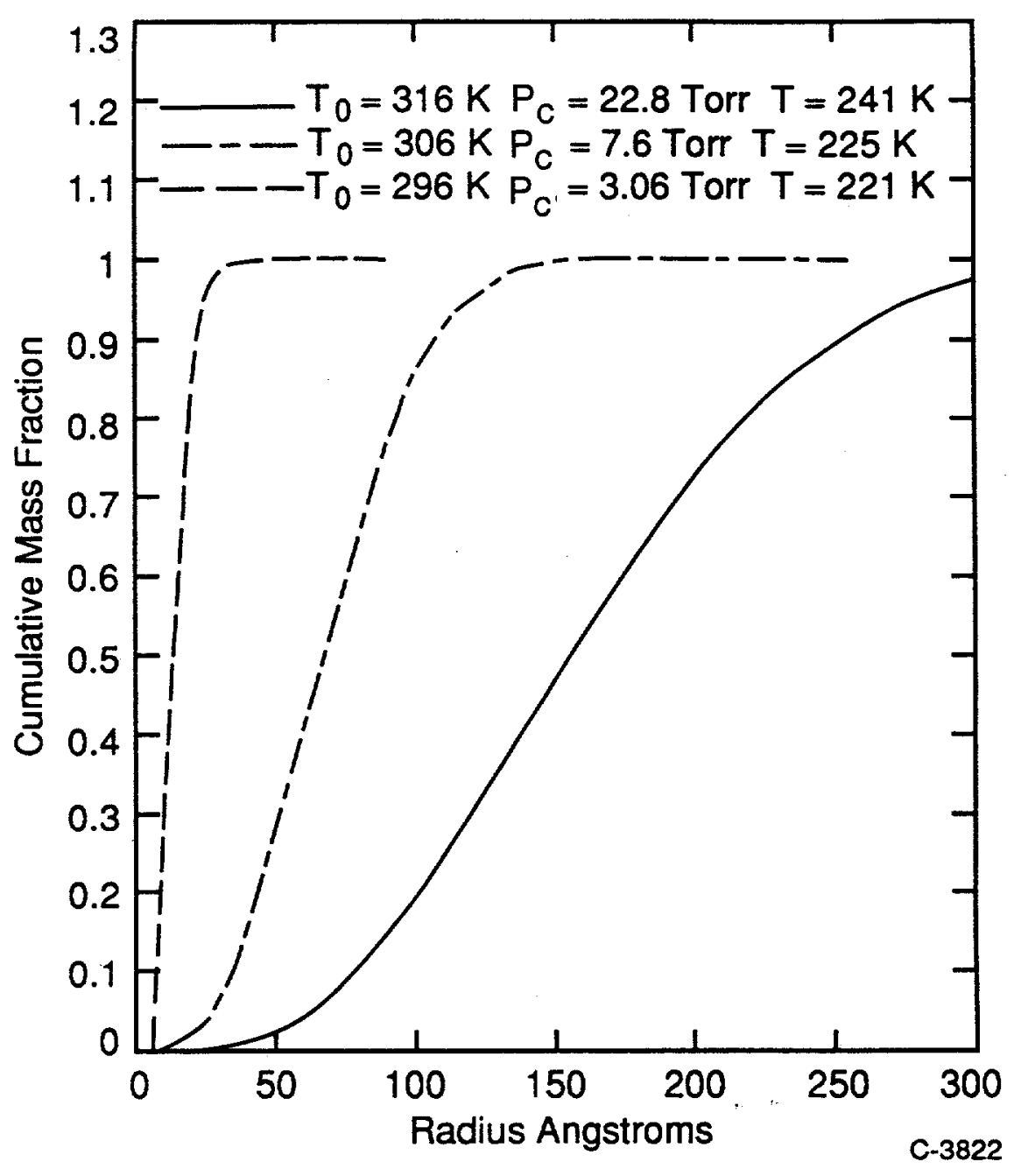

Figure 11. Cumulative Mass Distributions at Ethanol Condensation Onset for Low, ----, Intermediate, -- , and High, _-, Temperature Conditions. 


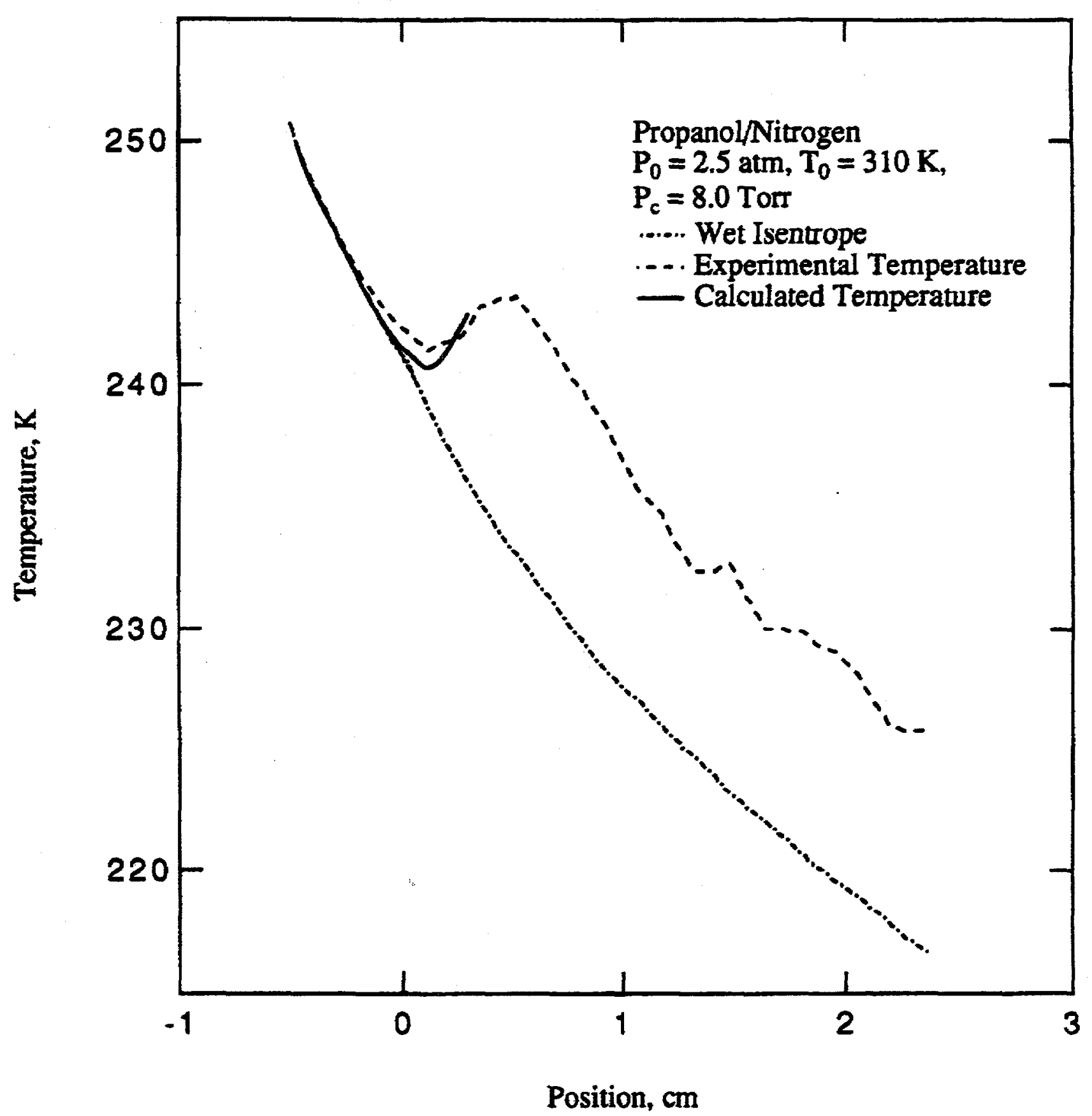

Figure 12. Experimental and Calculated Temperature Profiles for Propanol Condensation in the Supersonic Nozzle. $P_{c}=8$ Torr. 


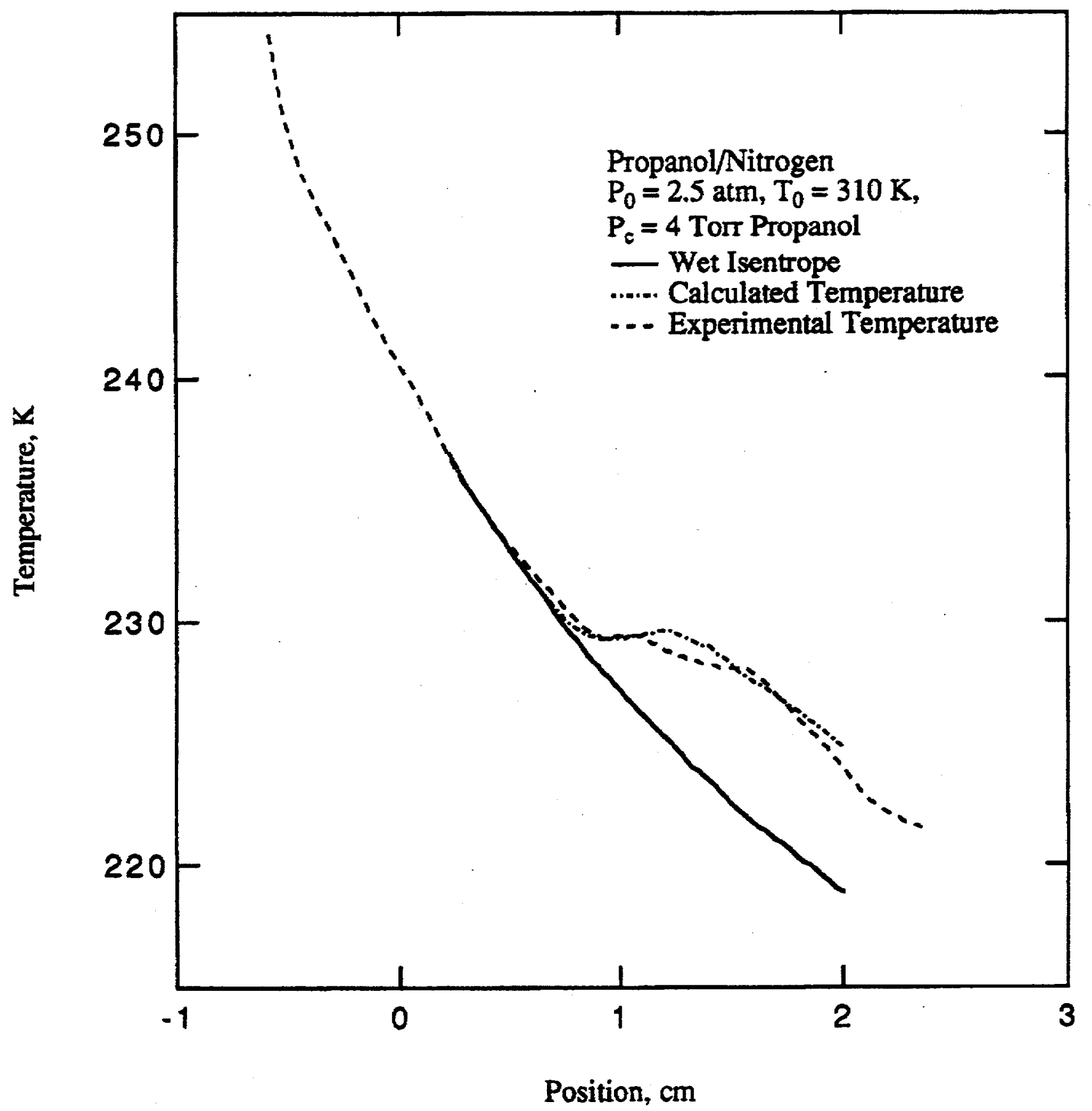

Figure 13. Experimental and Calculated Temperature Profiles for Propanol Condensation in the Supersonic Nozzle. $P_{c}=4$ Torr. 


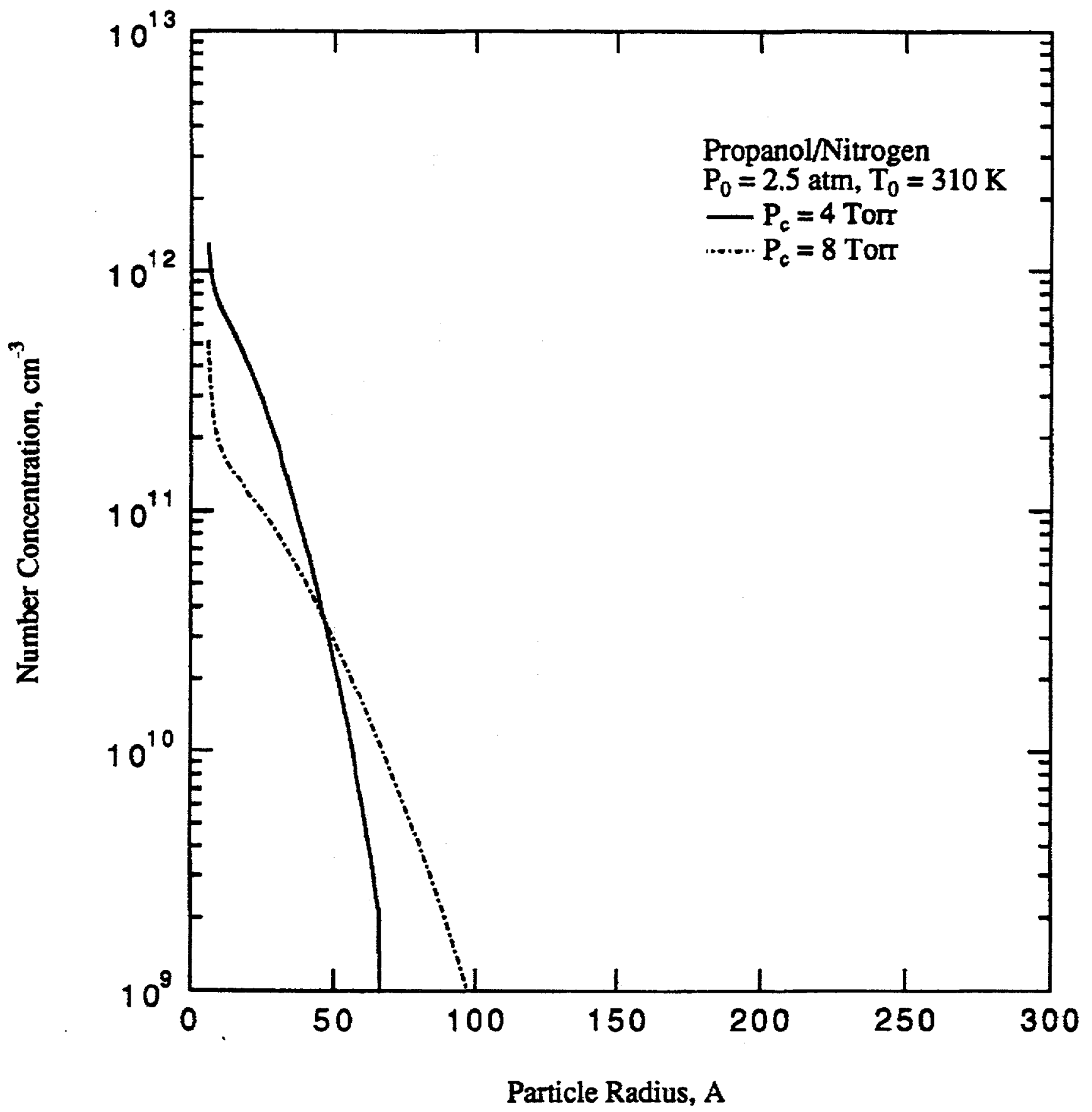

Figure 14. Propanol Droplet Size Distributions at Condensation Onset for Low and Intermediate Temperature Conditions of the Present Work. 


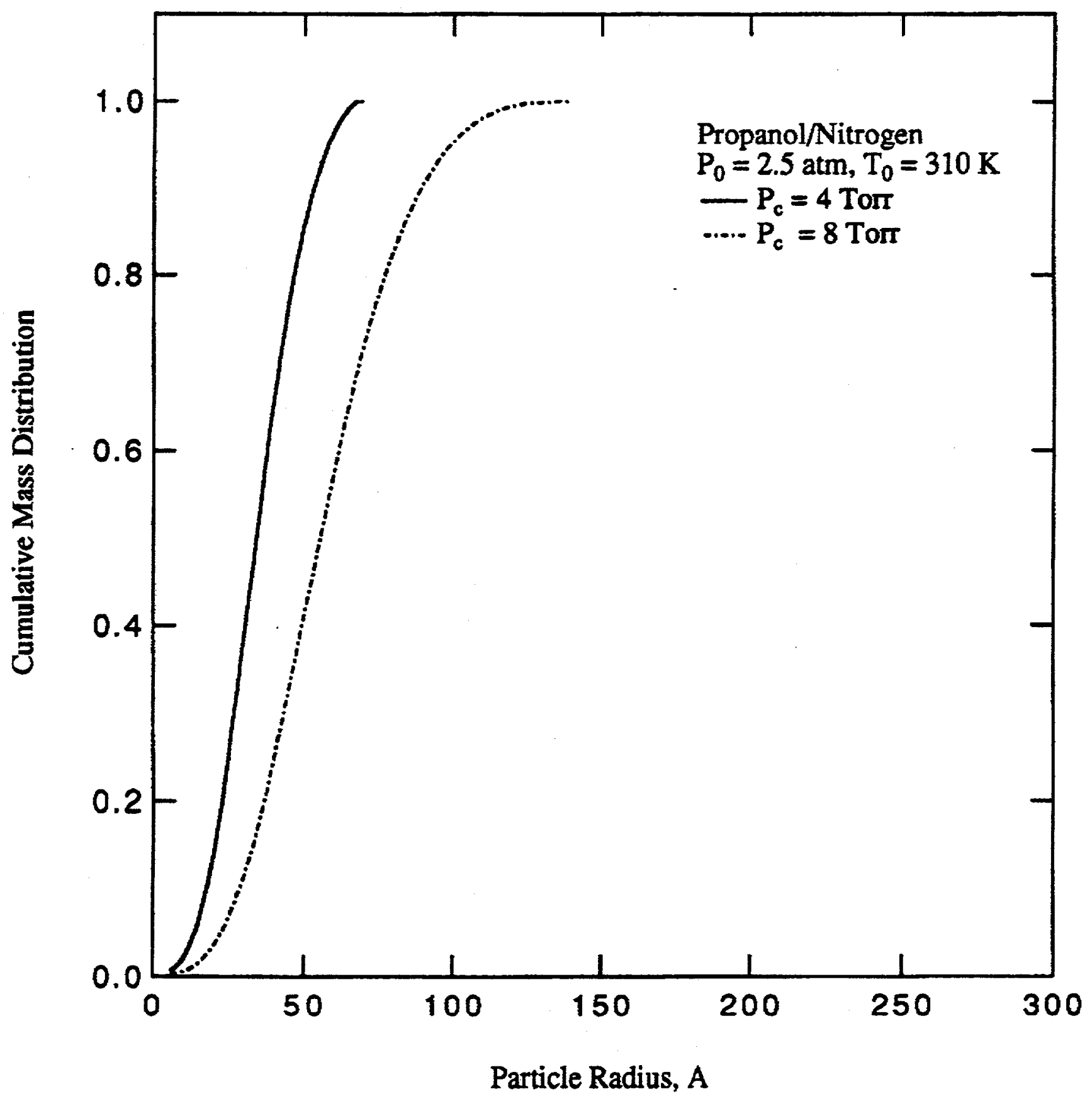

Figure 15. Propanol Cumulative Mass Distributions at Condensation Onset for Low and Intermediate Temperature Conditions of the Present Work. 